\title{
Development of vocal emotion recognition in school-age children: The EmoHI test for hearing-impaired populations
}

\author{
Leanne Nagels ${ }^{\text {Corresp., }, 2}{ }^{\text {, Etienne Gaudrain }}{ }^{2,3}$, Deborah Vickers ${ }^{4}$, Marta Matos Lopes ${ }^{5,6}$, Petra Hendriks ${ }^{1}$, Deniz \\ Başkent $^{2}$ \\ ${ }^{1}$ Center for Language and Cognition Groningen (CLCG), University of Groningen, Groningen, The Netherlands \\ 2 Department of Otorhinolaryngology/Head and Neck Surgery, University Medical Center Groningen, Groningen, The Netherlands \\ 3 CNRS, Lyon Neuroscience Research Center, Université de Lyon, Lyon, France \\ 4 Clinical Neurosciences Department, University of Cambridge, Cambridge, United Kingdom \\ 5 Hearbase Ltd, The Hearing Specialists, Kent, United Kingdom \\ 6 The Ear Institute, University College London, London, United Kingdom \\ Corresponding Author: Leanne Nagels \\ Email address: leanne.nagels@rug.nl
}

Traditionally, emotion recognition research has primarily used pictures and videos, while audio test materials are not always readily available or are not of good quality, which may be particularly important for studies with hearing-impaired listeners. Here we present a vocal emotion recognition test with pseudospeech productions from multiple speakers expressing three core emotions (happy, angry, and sad): the EmoHI test. Recorded with high sound quality, the test is suitable to use with populations of children and adults with normal or impaired hearing. Here we present normative data for vocal emotion recognition development in normal-hearing $(\mathrm{NH})$ school-age children using the $\mathrm{EmoHI}$ test. Furthermore, we investigated cross-language effects by testing NH Dutch and English children, and tested the suitability of using the EmoHI test with hearing-impaired populations by presenting preliminary data from prelingually deaf Dutch children with cochlear implants (Cls). Our results show that NH children's performance improved significantly with age from the youngest group tested on (4-6 years: $48.9 \%$, on average). However, NH children's performance did not reach adult-like values (adults: $94.1 \%$ ) even for the oldest age group tested (10-12 years: $81.1 \%$ ). Additionally, the effect of age on $\mathrm{NH}$ children's development did not differ across languages. All except one $\mathrm{Cl}$ child performed at or above chance-level showing the suitability of the EmoHI test. In addition, 7 out of 14 $\mathrm{Cl}$ children performed within the $\mathrm{NH}$ age-appropriate range, and even 9 out of $14 \mathrm{Cl}$ children did so when measuring from their age at $\mathrm{Cl}$ implantation (hearing age). However, $\mathrm{Cl}$ children showed great variability in their performance, ranging from ceiling $(97.2 \%)$ to below chance-level performance (27.8\%), which could not be explained by chronological age alone. The strong and consistent development in performance with age, the lack of 
significant differences across the tested languages for $\mathrm{NH}$ children, and the above-chance performance of most $\mathrm{Cl}$ children affirm the usability and versatility of the EmoHI test. 
1 Development of vocal emotion recognition in school-

2 age children: The EmoHI test for hearing-impaired

3 populations

4

5

6

7

8

Leanne Nagels ${ }^{1,2}$, Etienne Gaudrain ${ }^{3,2}$, Deborah Vickers ${ }^{4}$, Marta Matos Lopes ${ }^{5,6}$, Petra Hendriks $^{1}$, Deniz Başkent ${ }^{2}$

${ }^{1}$ Center for Language and Cognition Groningen (CLCG), University of Groningen, Groningen, The Netherlands

${ }^{2}$ Department of Otorhinolaryngology/Head and Neck Surgery, University Medical Center Groningen, Groningen, The Netherlands

${ }^{3}$ CNRS, Lyon Neuroscience Research Center, Université de Lyon, Lyon, France

${ }^{4}$ Clinical Neurosciences Department, University of Cambridge, Cambridge, UK

${ }^{5}$ Hearbase Ltd, The Hearing Specialists, Kent, UK

${ }^{6}$ The Ear Institute, University College London, London, UK

Corresponding Author: Leanne Nagels

Email address: leanne.nagels@rug.nl

\section{Abstract}

Traditionally, emotion recognition research has primarily used pictures and videos, while audio test materials are not always readily available or are not of good quality, which may be particularly important for studies with hearing-impaired listeners. Here we present a vocal emotion recognition test with pseudospeech productions from multiple speakers expressing three core emotions (happy, angry, and sad): the EmoHI test. Recorded with high sound quality, the test is suitable to use with populations of children and adults with normal or impaired hearing. Here we present normative data for vocal emotion recognition development in normal-hearing $(\mathrm{NH})$ school-age children using the EmoHI test. Furthermore, we investigated cross-language effects by testing NH Dutch and English children, and tested the suitability of using the EmoHI test with hearing-impaired populations by presenting preliminary data from prelingually deaf Dutch children with cochlear implants (CIs). Our results show that NH children's performance improved significantly with age from the youngest group tested on (4-6 years: $48.9 \%$, on average). However, NH children's performance did not reach adult-like values (adults: 94.1\%) even for the oldest age group tested (10-12 years: $81.1 \%$ ). Additionally, the effect of age on $\mathrm{NH}$ children's development did not differ across languages. All except one CI child performed at or above chance-level showing the suitability of the EmoHI test. In addition, 7 out of $14 \mathrm{CI}$ children performed within the $\mathrm{NH}$ age-appropriate range, and even 9 out of $14 \mathrm{CI}$ children did so when measuring from their age at $\mathrm{CI}$ implantation (hearing age). However, $\mathrm{CI}$ children showed great variability in their performance, ranging from ceiling (97.2\%) to below chance-level 
40 performance (27.8\%), which could not be explained by chronological age alone. The strong and 41 consistent development in performance with age, the lack of significant differences across the 42 tested languages for $\mathrm{NH}$ children, and the above-chance performance of most $\mathrm{CI}$ children affirm 43 the usability and versatility of the EmoHI test. 


\section{Introduction}

45 Development of emotion recognition in children has been studied extensively using visual

46

47

48

49

50

51

52

53

54

55

56

57

58

59

60

61

62

63

64

65

66

67

68

69

70

71

72

73

74

75

76

77

78

79

80

81

82

83 stimuli, such as pictures or sketches of facial expressions (e.g., Rodger et al., 2015), or audiovisual materials (e.g., Nelson \& Russell, 2011), and particularly in some clinical groups, such as autistic children (e.g., Harms, Martin \& Wallace, 2010). However, not much is known about the development of vocal emotion recognition, even in typically developing children (Scherer, 1986; Sauter, Panattoni \& Happé, 2013). While children can recognize facial and vocal emotions reliably and associate them with external causes already from the age of 5 years on (Pons, Harris \& de Rosnay, 2004), it seems to take until late childhood for this ability to develop to adult-like levels (Tonks et al., 2007; Sauter, Panattoni \& Happé, 2013). The recognition of vocal emotions relies heavily on the perception of related vocal acoustic cues, such as mean fundamental frequency (F0) and intensity, as well as fluctuations in these cues, and speaking rate (Scherer, 1986). Based on earlier research on the development of voice perception (Mann, Diamond \& Carey, 1979; Nittrouer \& Miller, 1997), children's performance may be lower compared to adults due to differences in their weighting of acoustic cues and a lack of robust representations of auditory categories. For instance, Morton and Trehub (2001) showed that when acoustic cues and linguistic content contradict the emotion they convey, children mostly rely on linguistic content to judge emotions, whereas adults mostly rely on affective prosody. In addition, children and adults both are better at facial emotion recognition than vocal emotion recognition (Nelson \& Russell, 2011; Chronaki et al., 2015). All of these observations combined indicate that the formation of robust representations for vocal emotions is highly complex and possibly a long-lasting process even in typically developing children.

Research with hearing-impaired children has shown that they do not perform as well on vocal emotion recognition compared to their normal-hearing $(\mathrm{NH})$ peers (Dyck et al., 2004; Hopyan-Misakyan et al., 2009; Nakata, Trehub \& Kanda, 2012; Chatterjee et al., 2015). HopyanMisakyan (2009) showed that 7-year-old children with cochlear implants (CIs) performed as well as their NH peers on visual emotion recognition but scored significantly lower on vocal emotion recognition. Visual emotion recognition generally seems to develop faster than vocal emotion recognition (Nowicki \& Duke, 1994; Nelson \& Russell, 2011), particularly in hearing-impaired children (Hopyan-Misakyan et al., 2009), which may indicate that visual emotion cues are perceptually more prominent or easier to categorize than vocal emotion cues. For hearingimpaired children, a higher reliance on visual emotion cues as compensation for spectrotemporally degraded auditory input may be an effective strategy, as emotion recognition in daily life is usually multimodal. However, it may lead to less robust auditory representations of vocal emotions and knowledge about their acoustic properties. Luo, Kern, and Pulling (2018) also showed that CI users' ability to recognize vocal emotions was related to their self-reported quality of life, which emphasizes the importance of recognizing vocal emotion cues in addition to visual emotion cues. Wiefferink et al. (2013) suggested that reduced auditory exposure and language delays may also lead to delayed social-emotional development and reduced conceptual knowledge about emotions, which in turn result in a negative impact on emotion recognition.

Peer) reviewing PDF | (2019:09:40928:2:0:NEW 4 Feb 2020) 
84 This is also evidenced by CI children's reduced differences in mean F0 cues and F0 variations in

85

86

87

88

89

90

91

92

93

94

95

96

97

98

99

100

101

102

103

104

105

106

107

108

109

110

111

112

113

114

115

116

117

118

119

120

121

122

123

emotion production compared to their NH peers (Chatterjee et al., 2019). The effects of conceptual knowledge on children's discrimination abilities have also been shown earlier, for instance, in research on pitch discrimination (Costa-Giomi \& Descombes, 1996). Costa-Giomi \& Descombes (1996) showed that French children showed better pitch discrimination when they had to use the single meaning terms 'aigu' and 'grave' to denote pitch than the multiple meaning words 'haut' [high] and 'bas' [low], which besides pitch also can be used to denote differences in space and loudness. This finding demonstrates that the label to refer to a concept may affect the labeling process itself. Thus, if CI children do not have clear conceptual knowledge about emotions, this will also similarly affect their ability to label them correctly. Finally, perceptual limitations, such as increased F0 discrimination thresholds (Deroche et al., 2014), may also play a role in CI children's abilities to recognize vocal emotions. Nakata, Trehub, and Kanda (2012) found that children with CIs especially had difficulties with differentiating happy from angry vocal emotions. This finding suggests that $\mathrm{CI}$ children primarily use speaking rate to categorize vocal emotions, as this cue differentiates sad from happy and angry vocal emotions but is similar for the latter two emotions. Therefore, hearing loss also seems to influence the weighting of different acoustic cues, and hence likely also affects the formation of representations of vocal emotions.

Vocal emotion recognition also differs from visual emotion recognition due to the potential influence of linguistic factors. Research regarding cross-language effects on emotion recognition has also demonstrated the importance of auditory exposure for vocal emotion recognition. Most studies have demonstrated a so-called 'native language benefit' showing that listeners are better at recognizing vocal emotions produced by speakers from their own native language than from another language. (Van Bezooijen, Otto \& Heenan, 1983; Scherer, Banse \& Wallbott, 2001; Bryant \& Barrett, 2008). This effect has been mainly attributed to cultural differences (Van Bezooijen, Otto \& Heenan, 1983), but also effects of language distance have been reported (Scherer, Banse \& Wallbott, 2001), i.e., differences in performance were larger when the linguistic distance (the extent to which the features of two languages differ from each other) between the speakers' and listeners' native languages was larger. Interestingly, Bryant and Barrett (2008) did not find a native language benefit for low-pass filtered vocal emotion stimuli, which filtered out both the linguistic message and the language-specific phonological information. Fleming et al. (2014) also demonstrated a similar native-language benefit for voice recognition based on differences in phonological familiarity. For CI children, reduced auditory exposure may also lead to reduced phonological familiarity, and therefore also contribute to difficulties with the recognition of vocal emotions.

As most research on the development of emotion recognition has used visual or audiovisual materials such as pictures or videos, good-quality audio materials are scarce. While the audio quality may only have a small effect on NH listeners' performance, it may be imperative for hearing-impaired listeners' vocal emotion recognition abilities. Hence, we recorded high sound quality vocal emotion recognition test stimuli produced by multiple

Peer) reviewing PDF | (2019:09:40928:2:0:NEW 4 Feb 2020) 
124 speakers with three basic emotions (happy, angry, and sad) that are suitable to use with hearing-

125

126

127

128

129

130

131

132

133

134

135

136

137

138

139

140

141

142

143

144

145

146

147

148

149

150

151

152

153

154

155

156

157

158

159

160

161

162

163

impaired children and adults: the EmoHI test. We aimed to investigate how NH school-age children's ability to recognize vocal emotions develops with age and to obtain normative data for the EmoHI test for future applications, for instance, with clinical populations. In addition, we tested children of two different native languages, namely Dutch and English, to investigate potential cross-language effects, and we collected preliminary data from Dutch prelingually deaf children with CIs, to investigate the applicability of the EmoHI test to hearing-impaired children.

\section{Materials \& Methods}

\section{Participants}

We collected normative data from fifty-eight Dutch and twenty-five English children between 4 and 12 years of age, and fifteen Dutch and fifteen English adults between 20 and 30 years of age with normal hearing. All NH participants were monolingual speakers of Dutch or English and reported no hearing or language disorders. Normal hearing (hearing thresholds at $20 \mathrm{~dB} \mathrm{HL}$ ) was screened with pure-tone audiometry at octave-frequencies between 500 and $4000 \mathrm{~Hz}$. In addition, we collected preliminary data from fourteen prelingually deaf Dutch children with CIs between 4 and 16 years of age. The study was approved by the Medical Ethical Review Committee of the University Medical Center Groningen (METc 2016.689). A written informed consent form was signed by adult participants and the parents or legal guardians of children before data collection.

\section{Stimuli and Apparatus}

We made recordings of six native Dutch speakers producing two non-language specific pseudospeech sentences using three core emotions (happy, sad, and angry), and a neutral emotion (not used in the current study). All speakers were native monolingual speakers of Dutch without any discernible regional accent and did not have any speech, language, or hearing disorders. Speakers gave written informed consent for the distribution and sharing of the recorded materials. To keep our stimuli relevant to emotion perception literature and suitable for usage across different languages, the pseudospeech sentences that we used, Koun se mina lod

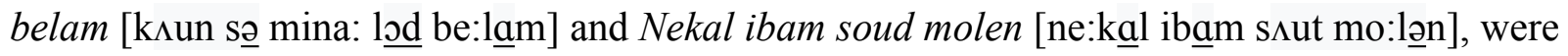
based on the Geneva Multimodal Emotion Portrayal (GEMEP) Corpus materials by Bänziger, Mortillaro \& Scherer (2012). These pseudosentences are meaningful neither in Dutch nor in English, nor in any other Indo-European languages. Speakers were instructed to produce the sentences in a happy, sad, angry, or neutral manner using emotional scripts that were also used for the GEMEP corpus stimuli (Scherer \& Bänziger, 2010). We chose these three core emotions as previous studies have reported that children first learn to identify happy, angry, and sad emotions, respectively, followed by fear, surprise, and disgust (Widen \& Russell, 2003), and hence we could test children from very young ages. The stimuli were recorded in an anechoic room at a sampling rate of $44.1 \mathrm{kHz}$.

We pre-selected 96 productions, including neutral productions, (2 productions $\times 2$ sentences x 4 emotions x 6 speakers) and performed a short online survey with Dutch and 
164 English adults to confirm that the stimuli were recognized reliably and to select the four speakers

165

166

167

168

169

170

171

172

173

174

175

176

177

178

179

180

181

182

183

184

185

186

187

188

189

190

191

192

193

194

195

196

197

198

199

200

201

202

203

whose productions were recognized best. Table 1 shows an overview of these four selected speakers' demographic information and voice characteristics. The neutral productions and the productions of the other two speakers were part of the online survey, and are available with the stimulus set, but were not used in the current study to simplify the task for children. Our final set of stimuli consisted of 36 experimental stimuli with three productions (one sentence repeated once + the other sentence) per emotion and per speaker ( 3 productions $\mathrm{x} 3$ emotions $\mathrm{x} 4$ speakers) as well as 4 practice stimuli with one production per speaker that were used for the training session.

$<$ insert Table 1>

\section{Procedure}

$\mathrm{NH}$ and $\mathrm{CI}$ children were tested in a quiet room at their home, and $\mathrm{NH}$ adults were tested in a quiet testing room at the two universities. Since the present experiment was part of a larger project on voice and speech perception (Perception of Indexical Cues in Kids and Adults (PICKA)), data were collected from the same population of children and adults in multiple experiments, see, for instance, Nagels et al. (in review). The experiment started with a training session consisting of 4 practice stimuli and was followed by the test session consisting of 36 experimental stimuli. The total duration of the experiment was approximately 6 to 8 minutes. All stimuli were presented to participants in a randomized order.

The experiment was conducted on a laptop with a touchscreen using a child-friendly interface that was developed in Matlab (Fig. 1). The auditory stimuli were presented via Sennheiser HD 380 Pro headphones for NH children and adults, and via Logitech Z200 loudspeakers for CI children. The presentation level of the stimuli was calibrated to a sound level of $65 \mathrm{dBA}$. CI children were instructed to use the settings they most commonly use in daily life and to keep the settings consistent throughout the experiment. In each trial, participants heard a stimulus and then had to indicate which emotion was conveyed by clicking on one of three corresponding clowns on the screen. Visual feedback on the accuracy of responses was provided to motivate participants. Participants saw confetti falling down the screen after a correct response, and the parrot shaking its head after an incorrect response. After every two trials, one of the clowns in the back went one step up the ladder until the experiment was finished to keep children engaged and to give an indication of the progress of the experiment.

$<$ insert Figure 1 $>$

\section{Data analysis}

NH children's accuracy scores were analyzed using the lme4 package (Bates et al., 2014) in R. A mixed-effects logistic regression model with a three-way interaction between language (Dutch and English), emotion (happy, angry, and sad), and age in decimal years, and random intercepts 
204 per participant and per stimulus was computed to determine the effects of language, emotion, and 205 age on NH children's ability to recognize vocal emotions. We used backward stepwise selection 206 with ANOVA Chi-Square tests to select the best fitting model, starting with the full factorial 207 model, in lme4 syntax: accuracy language * emotion * age + (1|participant) + 208 (1|stimulus), and deleting one fixed factor at a time based on its significance. In addition, we 209 performed Dunnett's tests on the NH Dutch and English data with accuracy as an outcome 210 variable and age group as a predictor variable using the DescTools package (Signorell et al., 211 2018) to investigate at what age NH Dutch and English children show adult-like performance. 212 Finally, we examined our preliminary data of CI children to investigate if they could reliably 213 perform the task.

214

215 Results

216 NH Dutch and English data

217 Figure 2 shows the accuracy scores of NH Dutch (left panel) and English (right panel)

218 participants as a function of their age (dots) and age group (boxplots). Model comparison

219 showed that the full model with random intercepts per participant and per stimulus was 220 significantly better than the full models with only random intercepts per participant $\left[\chi^{2}(1)=393\right.$, $221 p<0.001]$ or only random intercepts per stimulus $\left[\chi^{2}(1)=51.9, p<0.001\right]$. Backward stepwise 222 selection showed that the best fitting and most parsimonious model was the model with only a 223 fixed effect of age, in lme4 syntax: accuracy age + (1|participant) + (1|stimulus). This

224

225

226

227

228

229

230

231

232

233

234

235

236

237

238

239

240

241 model did not significantly differ from the full model $\left[\chi^{2}(10)=12.90, p=0.23\right]$ or any of the other models while being the most parsimonious. Figure 2 shows the data of individual participants and the median accuracy scores per age group for the NH Dutch and English participants. NH children's ability to recognize vocal emotions correctly significantly increased as a function of age [z-value $=8.91$, estimate $=0.30, \mathrm{SE}=0.034, p<0.001]$. We did not find any significant effects of language or emotion on children's accuracy scores. Finally, the results of the Dunnett's tests showed that the accuracy scores of Dutch NH children of all tested age groups differed from Dutch NH adults [4-6 years difference $=-0.47, p<0.001 ; 6-8$ years difference $=-0.31, p<0.001 ; 8-10$ years difference $=-0.19, p<0.001 ; 10-12$ years difference $=$ $0.15, p<0.001]$, and the accuracy scores of English NH children of all tested age groups differed from English NH adults [4-6 years difference $=-0.43, p<0.001 ; 6-8$ years difference $=-0.27, p$ $<0.001 ; 8-10$ years difference $=-0.20, p<0.001 ; 10-12$ years difference $=-0.12, p<0.01]$. The mean accuracy scores per age group and language are shown in Table 2.

$<$ insert Figure 1 $>$

$<$ insert Table 2> 


\section{Preliminary data of $\mathrm{Cl}$ children}

243 Figure 3 shows the accuracy scores of Dutch CI children as a function of their chronological age

244 (left panel) and hearing age (right panel), the latter based on the age at which they received the

245 CI. The mean accuracy scores per age group are shown in Table 2. All except one CI child

246 performed at or above chance-level. Based on Figure 3, we can see that 7 out of 14 CI children

247 (50\%) performed within the $\mathrm{NH}$ age-appropriate range. Considering CI children's hearing age

248 instead of their chronological age, 9 out of $14 \mathrm{CI}$ children (64.3\%) still show performance within

249 the NH age-appropriate range. However, there is a large variability in CI children's performance,

250 which varies from ceiling (97.2\%) to below chance-level performance (27.8\%). The

251 development in CI children's performance with age does not seem to be as consistent as we

252 found for $\mathrm{NH}$ children, which suggests that their performance is not merely due to age-related

253 development.

254

255

$<$ insert Figure 3 $>$

256

257 Discussion

\section{Age effect}

259 As shown by our results and the data displayed in Figure 2, NH children's ability to recognize 260 vocal emotions improved gradually as a function of age. In addition, we found that, on average, 261 even the oldest age group of 10- to 12-year-old Dutch and English children did not show adult262 like performance yet. The 4-year-old NH children that were tested performed at or above chance 263 level while adults generally showed near ceiling performance, indicating that our test covers a 264 wide range of age-related performances. Our results are in line with previous findings that $\mathrm{NH}$ 265 children's ability to recognize vocal emotions improves gradually as a function of age (Tonks et 266 al., 2007; Sauter, Panattoni \& Happé, 2013). It may be that children require more auditory 267 experience to form robust representations of vocal emotions or rely on different acoustic cues 268 than adults, as was shown in research on the development of sensitivity to voice cues (Mann, 269 Diamond \& Carey, 1979; Nittrouer \& Miller, 1997). It is still unclear on which specific acoustic 270 cues children are basing their decisions and how this differs from adults. Future research using 271 machine-learning approaches may be able to further explore such aspects. Finally, the visual 272 feedback may have caused some learning effects, although the correct response was not shown 273 after an error, and learning would pose relatively high demands on auditory working memory 274 since there were only three productions per speaker and per emotion presented in a randomized 275 order.

276

\section{Language effect}

278 Comparing data from NH children from two different native languages, we did not find any 279 cross-language effects between Dutch and English children's development of vocal emotion 280 recognition, even though the materials were produced by Dutch native speakers. Earlier research 281 has demonstrated that although adults are able to recognize vocal emotions across languages, 
282 there still seems to be a native language benefit (Van Bezooijen, Otto \& Heenan, 1983; Scherer, 283 Banse \& Wallbott, 2001; Bryant \& Barrett, 2008). Listeners were better at recognizing vocal 284 emotions that were produced by speakers of their native language than another language.

285 However, it should be noted that five (Scherer, Banse \& Wallbott, 2001; Bryant \& Barrett, 2008) 286 and nine (Van Bezooijen, Otto \& Heenan, 1983) different and more complex emotions were used 287 in these studies which likely poses a considerably more difficult task than differentiating three 288 basic emotions. In addition, the lack of a native language benefit in our results may also be due 289 to the fact that Dutch and English are phonologically closely related languages. This idea is also 290 in line with the language distance effect (Scherer, Banse \& Wallbott, 2001) and phonological 291 familiarity effects (Bryant \& Barrett, 2008). We are currently collecting data from Turkish 292 children and adults to investigate whether there are any detectable cross-language effects for 293 typologically and phonologically more distinct languages.

294

295

296

297

298

299

300

301

302

303

304

305

306

307

308

309

310

311

312

313

314

315

316

317

318

\section{Conclusions}

320 The results of the current study provide baseline normative data for the development of vocal 321 emotion recognition in typically developing, school-age children with normal hearing using the 
322 EmoHI test. Our results show that there is a large but relatively slow and consistent development 323 in children's ability to recognize vocal emotions. Furthermore, the preliminary data from the CI 324 children show that they seem to be able to carry out the EmoHI test reliably, but the 325 improvement in their performance as a function of age was not as consistent as for NH children. 326 The clear development observed in NH children's performance as a function of age and the 327 generalizability of performance across the tested languages show the EmoHI test's suitability for 328 different ages and potentially also across different languages. Additionally, the above-chance 329 performance of most CI children and the high sound quality stimuli also demonstrate that the 330 EmoHI test is suitable for testing hearing-impaired populations. 


\section{Acknowledgments}

332 We are grateful to all of the children, parents, and students that took part in the study, the

333

334

335

336

337

338

339

340

341

342

343

344

345

346

347

348

349

350

351

352

353

354

355

356

357

358

359

360

361

362

363

364

365

366

367

368

369

370

speakers that were recorded for our stimuli, and Basisschool de Brink in Ottersum, Basisschool de Petteflet, and BSO Huis de B in Groningen for their help with recruiting NH child participants and the clinic of the Otorhinolaryngology Department of the University Medical Center Groningen, particularly ir. Bert Maat and Dr. Rolien Free, for their help with the recruitment of CI child participants. We would also like to thank Iris van Bommel, Evelien Birza, Paolo Toffanin, Jacqueline Libert, Jemima Phillpot, and Jop Luberti (illustrations) for their contribution to the development of the game interfaces, and Monita Chatterjee for her advice on recording the sound stimuli. Finally, we would like to thank the Vocal Interactivity in-and-between Humans, Animals, and Robots (VIHAR) workshop committee for awarding our proceedings paper with the PeerJ best contribution award which resulted in the current paper.

\section{References}

Bänziger T, Mortillaro M, Scherer KR. 2012. Introducing the Geneva Multimodal expression corpus for experimental research on emotion perception. Emotion 12:1161-1179. DOI: 10.1037/a0025827.

Bates D, Maechler M, Bolker B, Walker S, Christensen RHB, Singmann H, Dai B, Grothendieck G. 2014. Package 'Ime4.' R Foundation for Statistical Computing, Vienna 12.

Bryant G, Barrett HC. 2008. Vocal emotion recognition across disparate cultures. Journal of Cognition and Culture 8:135-148. DOI: 10.1163/156770908X289242.

Chatterjee M, Kulkarni AM, Siddiqui RM, Christensen JA, Hozan M, Sis JL, Damm SA. 2019. Acoustics of emotional prosody produced by prelingually deaf children with cochlear implants. Frontiers in Psychology 10:1-15. DOI: 10.3389/fpsyg.2019.02190.

Chatterjee M, Zion DJ, Deroche ML, Burianek BA, Limb CJ, Goren AP, Kulkarni AM, Christensen JA. 2015. Voice emotion recognition by cochlear-implanted children and their normally-hearing peers. Hearing Research 322:151-162. DOI: 10.1016/j.heares.2014.10.003.

Chronaki G, Hadwin JA, Garner M, Maurage P, Sonuga-Barke EJS. 2015. The development of emotion recognition from facial expressions and non-linguistic vocalizations during childhood. British Journal of Developmental Psychology 33:218-236. DOI: 10.1111/bjdp.12075.

Costa-Giomi E, Descombes V. 1996. Pitch labels with single and multiple meanings: A study with French-speaking children. Journal of Research in Music Education 44:204-214. DOI: $10.2307 / 3345594$.

Deroche MLD, Lu H-P, Limb CJ, Lin Y-S, Chatterjee M. 2014. Deficits in the pitch sensitivity of cochlear-implanted children speaking English or Mandarin. Frontiers in Neuroscience 8:1-13. DOI: 10.3389/fnins.2014.00282.

Dyck MJ, Farrugia C, Shochet IM, Holmes-Brown M. 2004. Emotion recognition/understanding ability in hearing or vision-impaired children: do sounds, sights, or words make the

Peer) reviewing PDF | (2019:09:40928:2:0:NEW 4 Feb 2020) 
371

372

373

374

375

376

377

378

379

380

381

382

383

384

385

386

387

388

389

390

391

392

393

394

395

396

397

398

399

400

401

402

403

404

405

406

407

408

difference? Journal of Child Psychology and Psychiatry 45:789-800. DOI:

10.1111/j.1469-7610.2004.00272.x.

Fleming D, Giordano BL, Caldara R, Belin P. 2014. A language-familiarity effect for speaker discrimination without comprehension. Proceedings of the National Academy of Sciences 111:13795-13798. DOI: 10.1073/pnas.1401383111.

Harms MB, Martin A, Wallace GL. 2010. Facial emotion recognition in autism spectrum disorders: a review of behavioral and neuroimaging studies. Neuropsychology Review 20:290-322. DOI: 10.1007/s11065-010-9138-6.

Hopyan-Misakyan TM, Gordon KA, Dennis M, Papsin BC. 2009. Recognition of affective speech prosody and facial affect in deaf children with unilateral right cochlear implants. Child Neuropsychology 15:136-146. DOI: 10.1080/09297040802403682.

Luo X, Kern A, Pulling KR. 2018. Vocal emotion recognition performance predicts the quality of life in adult cochlear implant users. The Journal of the Acoustical Society of America 144:EL429-EL435. DOI: 10.1121/1.5079575.

Mann VA, Diamond R, Carey S. 1979. Development of voice recognition: Parallels with face recognition. Journal of Experimental Child Psychology 27:153-165. DOI: 10.1016/00220965(79)90067-5.

Morton JB, Trehub SE. 2001. Children's understanding of emotion in speech. Child Development 72:834-843. DOI: 10.1111/1467-8624.00318.

Nagels L, Gaudrain E, Vickers D, Hendriks P, Başkent D. (in review). Development of voice perception is dissociated across gender cues in school-age children.

Nakata T, Trehub SE, Kanda Y. 2012. Effect of cochlear implants on children's perception and production of speech prosody. The Journal of the Acoustical Society of America 131:1307-1314. DOI: 10.1121/1.3672697.

Nelson NL, Russell JA. 2011. Preschoolers' use of dynamic facial, bodily, and vocal cues to emotion. Journal of Experimental Child Psychology 110:52-61. DOI: 10.1016/j.jecp.2011.03.014.

Nittrouer S, Miller ME. 1997. Predicting developmental shifts in perceptual weighting schemes. The Journal of the Acoustical Society of America 101:2253-2266. DOI: 10.1121/1.418207.

Nowicki S, Duke MP. 1994. Individual differences in the nonverbal communication of affect: The diagnostic analysis of nonverbal accuracy scale. Journal of Nonverbal Behavior 18:9-35. DOI: 10.1007/BF02169077.

Pons F, Harris PL, de Rosnay M. 2004. Emotion comprehension between 3 and 11 years: Developmental periods and hierarchical organization. European journal of developmental psychology 1:127-152. DOI: 10.1080/17405620344000022.

Rodger H, Vizioli L, Ouyang X, Caldara R. 2015. Mapping the development of facial expression recognition. Developmental Science 18:926-939. DOI: 10.1111/desc.12281. 
409 Sauter DA, Panattoni C, Happé F. 2013. Children's recognition of emotions from vocal cues.

410 British Journal of Developmental Psychology 31:97-113. DOI: 10.1111/j.2044-

411 835X.2012.02081.x.

412 Scherer KR. 1986. Vocal affect expression: A review and a model for future research.

413 Psychological Bulletin 99:143-165. DOI: 10.1037/0033-2909.99.2.143.

414 Scherer KR, Banse R, Wallbott HG. 2001. Emotion inferences from vocal expression correlate 415 across languages and cultures. Journal of Cross-Cultural Psychology 32:76-92. DOI:

$416 \quad 10.1177 / 0022022101032001009$.

417 Scherer KR, Bänziger T. 2010. On the use of actor portrayals in research on emotional

418 expression. In: Blueprint for affective computing: A sourcebook. 271-294.

419

420

421

Signorell A, Aho K, Alfons A, Anderegg N, Aragon T. 2018. DescTools: Tools for descriptive statistics. R package version 0.99.25. R Found. Stat. Comput., Vienna, Austria.

422

423

424

425

426

427

428

429

Tonks J, Williams WH, Frampton I, Yates P, Slater A. 2007. Assessing emotion recognition in 9-15-years olds: Preliminary analysis of abilities in reading emotion from faces, voices and eyes. Brain Injury 21:623-629. DOI: 10.1080/02699050701426865.

Van Bezooijen R, Otto SA, Heenan TA. 1983. Recognition of vocal expressions of emotion: A three-nation study to identify universal characteristics. Journal of Cross-Cultural Psychology 14:387-406. DOI: 10.1177/0022002183014004001.

Widen SC, Russell JA. 2003. A closer look at preschoolers' freely produced labels for facial expressions. Developmental Psychology 39:114-128. DOI: 10.1037/0012-1649.39.1.114.

Wiefferink CH, Rieffe C, Ketelaar L, De Raeve L, Frijns JHM. 2013. Emotion understanding in 430 deaf children with a cochlear Implant. Journal of Deaf Studies and Deaf Education

431 18:175-186. DOI: 10.1093/deafed/ens042. 
Figure 1

The experiment interface of the EmoHI test.

The illustrations were made by Jop Luberti. This image is published under the CC BY NC 4.0 license (https://creativecommons.org/licenses/by-nc/4.0/).

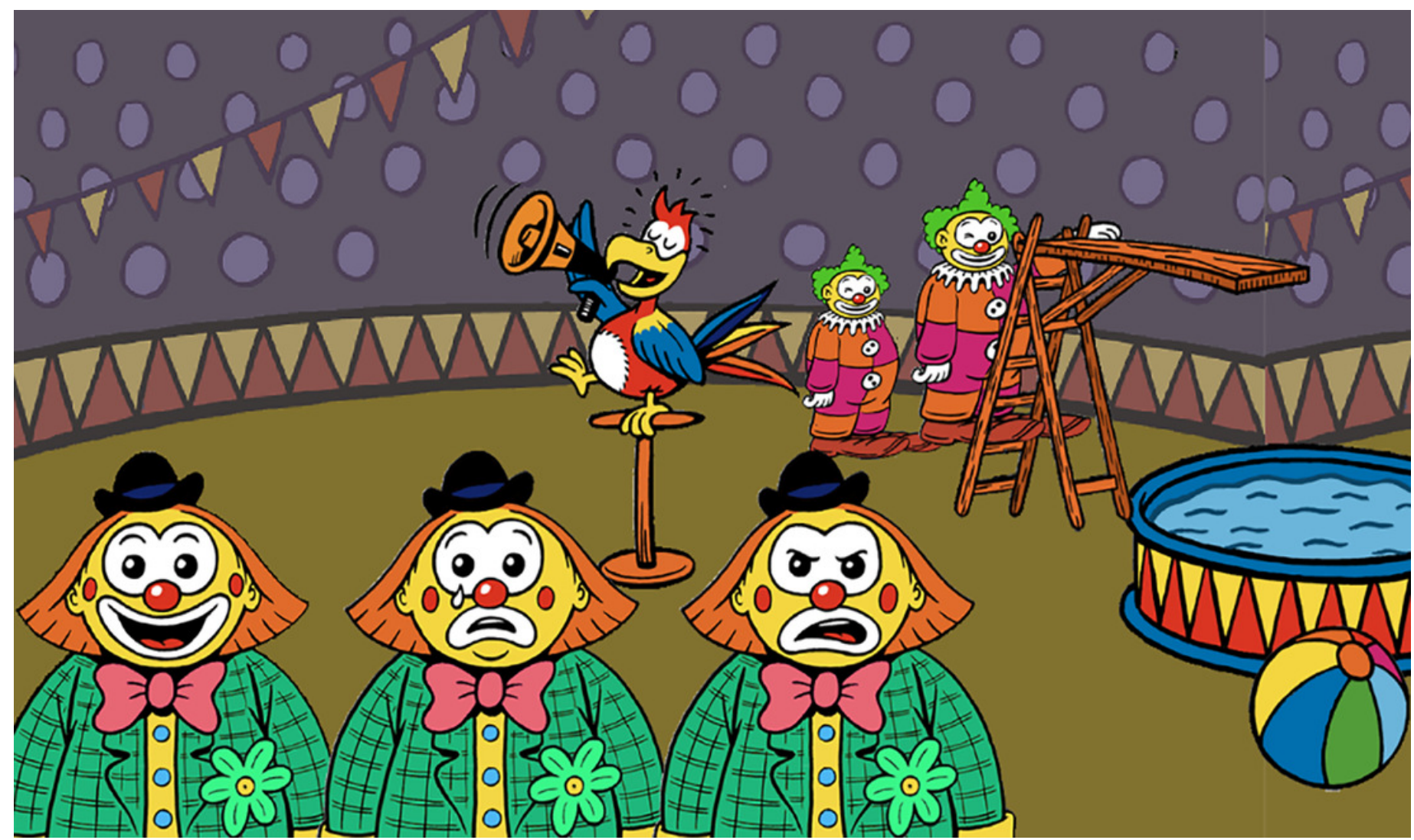




\section{Figure 2}

Emotion recognition in $\mathrm{NH}$ children and adults.

Accuracy scores of NH Dutch and English children and adults for the EmoHI test per age group and per language (Dutch in the left panel; English in the right panel). Pitch Labels with Single and Multiple Meanings: A Study with French-Speaking Children The dotted line shows the chance-level performance of $33.3 \%$ correct. The dots show individual data points at participants' age (Netherlands (NL): $\mathrm{N}_{\text {chidren }}=58, \mathrm{~N}_{\text {adults }}=15$; United Kingdom (UK) : $\mathrm{N}_{\text {children }}=$ $25, N_{\text {adults }}=15$ ). The boxplots show the median accuracy scores per age group, and the lower and upper quartiles. The whiskers indicate the lowest and highest data points within plus or minus 1.5 times the interquartile range.

\section{Emotion recognition in $\mathrm{NH}$ children and adults}

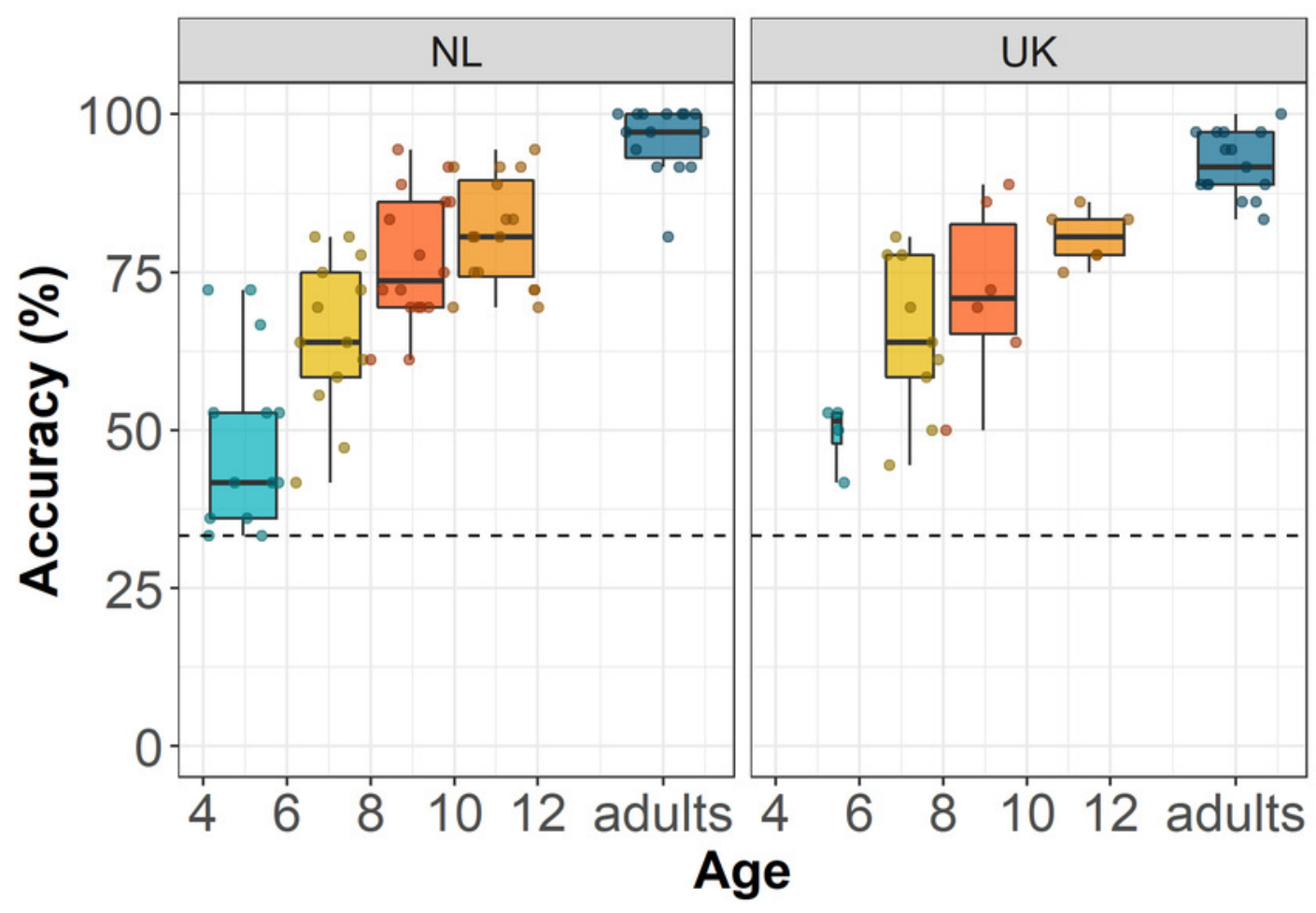

Age groups 追 4-6 years 帛 6-8 years 8-10 years 追 10-12 years 追 adults 


\section{Figure 3}

Emotion recognition in Dutch $\mathrm{Cl}$ children.

Accuracy scores of Dutch $\mathrm{Cl}$ children $(\mathrm{N}=14)$ for the EmoHI test per age group. Pitch Labels with Single and Multiple Meanings: A Study with French-Speaking Children The dotted line shows the chance-level performance of $33.3 \%$ correct. The dots show individual data points at Dutch $\mathrm{Cl}$ children's chronological age (left panel) and at their hearing age (right panel). The boxplots show NH Dutch children's median accuracy scores per age group, and the lower and upper quartiles, reproduced from Figure 2 . The whiskers indicate the lowest and highest data points of $\mathrm{NH}$ Dutch children within plus or minus 1.5 times the interquartile range.

\section{Emotion recognition in Dutch $\mathrm{Cl}$ children}
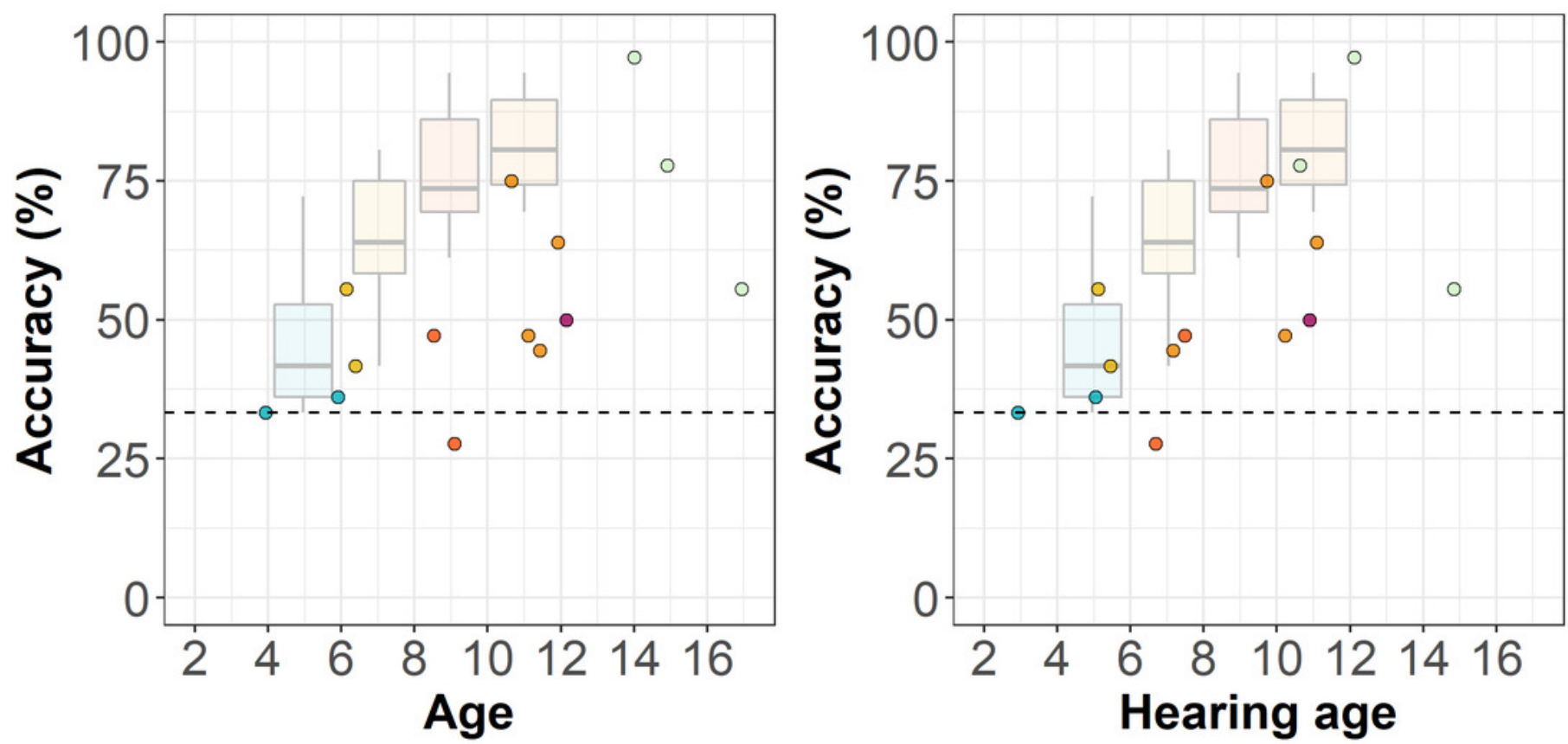

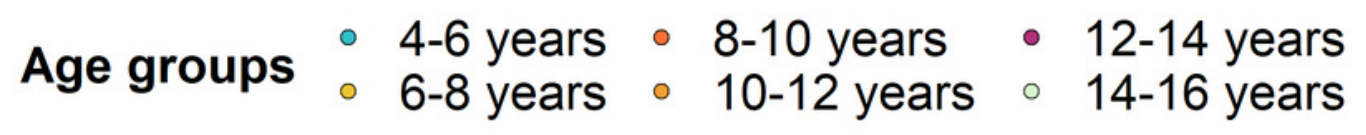


Table $\mathbf{1}$ (on next page)

Overview of the speakers' voice characteristics. 
1

Table 1: Overview of the speakers' voice characteristics.

2

\begin{tabular}{cccccc}
\hline Speaker & Age (years) & Gender & Height $(\mathbf{m})$ & Mean F0 $(\mathbf{H z})$ & F0 range $(\mathbf{H z})$ \\
\hline T2 & 36 & F & 1.68 & 302.23 & $200.71-437.38$ \\
T3 & 27 & M & 1.85 & 166.92 & $100.99-296.47$ \\
T5 & 25 & F & 1.63 & 282.89 & $199.49-429.38$ \\
T6 & 24 & M & 1.75 & 167.76 & $87.46-285.79$ \\
\hline
\end{tabular}

3 
Table 2 (on next page)

Overview of the mean accuracy scores (\%) per participant and age group. 
1

Table 2: Overview of the mean accuracy scores (\%) per participant and age group.

2

\begin{tabular}{cccc}
\hline Age groups & \multicolumn{3}{c}{ Participant groups } \\
\hline & Dutch $N H$ & English $N H$ & Dutch CI \\
\hline 4-6 years & $48.7 \%$ & $49.3 \%$ & $34.7 \%$ \\
6-8 years & $65.2 \%$ & $64.8 \%$ & $48.6 \%$ \\
8-10 years & $76.7 \%$ & $71.8 \%$ & $37.5 \%$ \\
10-12 years & $81.2 \%$ & $80.6 \%$ & $57.6 \%$ \\
12-14 years & - & - & $50.0 \%$ \\
14-16 years & - & - & $76.9 \%$ \\
Adults & $96.1 \%$ & $92.0 \%$ & - \\
\hline
\end{tabular}

3 